\title{
ISOPERIMETRIC INEQUALITIES FOR A NONLINEAR EIGENVALUE PROBLEM
}

\author{
CATHERINE BANDLE
}

\begin{abstract}
An estimate for the spectrum of the two-dimensional eigenvalue problem $\Delta u+\lambda e^{u}=0$ in $D(\lambda>0), u=0$ on $\partial D$ is derived, and upper and lower pointwise bounds for the solutions are constructed.
\end{abstract}

1. Let $D$ be a simply connected bounded domain in the plane with a piecewise analytic boundary $\partial D$. Consider the nonlinear Dirichlet problem

$$
\begin{aligned}
\Delta u(x)+\lambda e^{u(x)} & =0 & & \text { in } D, \\
u(x) & =0 & & \text { on } \partial D,
\end{aligned}
$$

where $\lambda$ is a positive real number and $x$ stands for the generic point $\left(x_{1}, x_{2}\right)$. This problem arises in the theory of self-ignition of a chemically active gas [4 and the literature cited there] and has been studied by many authors [2], [3], [5].

It was shown in [3] and [5] that there exists a number $\lambda^{*}>0$ such that the problem has at least one solution for each $\lambda \leqslant \lambda^{*}$, but does not have solutions for $\lambda>\lambda^{*}$. Bounds for $\lambda^{*}$ are found in [2]. In particular it was proved that $\lambda^{*} \geqslant 2 \pi / A$ where $A$ denotes the area of $D$. Equality is attained if and only if $D$ is a circle. In this paper we prove that $\lambda^{*} \leqslant 2 / R_{0}^{2}, R_{0}$ being the maximal conformal radius of $D$. We also give estimates for the solutions by means of the conformal radius. Our proofs are based on the introduction of a special system of coordinates defined by the level lines; see [6].

2. Let $g(x, \xi)$ be the Green's function for the Laplace operator, vanishing on $\partial D$. It is well known that for fixed $x \in D$

$$
g(x, \xi)=(2 \pi)^{-1} \log \left(R_{x} /|x-\xi|\right)+H(x, \xi)
$$

where $R_{x}$ is the conformal radius of $x$ with respect to $D$,

$$
|x-\xi|=\left(\sum_{i=1}^{2}\left(x_{i}-\xi_{i}\right)^{2}\right)^{1 / 2}
$$

and $H(x, \xi)$ is a harmonic function of $\xi$ with $\lim _{\xi \rightarrow x} H(x, \xi)=0$. With the help of this Green's function Problem (1) can be written as an integral equation:

Received by the editors November 18, 1974 and, in revised form, May 29, 1975.

AMS (MOS) subject classifications (1970). Primary 35B45, 52A40.

- American Mathematical Society 1976 


$$
u(x)=\lambda \int_{D} g(x, \xi) e^{u(\xi)} d \xi, \quad \text { where } d \xi=d \xi_{1} d \xi_{2} .
$$

We now keep $x$ fixed and denote by $D(t)$ the domain $\{\xi \in D ; g(x, \xi)>t\}$. It is homeomorphic to a circle. Let us assume that Problem (1) has a solution $u \in C^{2}(D) \cap C^{0}(\bar{D})$. Since $e^{t}$ is real analytic, $u(x)$ is also real analytic. Define

$$
a(t)=\int_{D(t)} e^{u(\xi)} d \xi
$$

Let $\Gamma(t)=\{\xi \in D ; g(x, \xi)=t\}$. It is a simple closed curve and analytic for all $t>0$. Denote by $\delta n>0$ the piece of normal between $\Gamma(t)$ and $\Gamma(t+d t)$. If $s$ is the arclength of $\Gamma(t)$, then [6, especially p. 213]

$$
d a(t) \equiv a(t+d t)-a(t)=-\oint_{\Gamma(t)} e^{u(\xi)} \delta n d s_{\xi}+o(d t) .
$$

Because of the strong maximum principle, $|\operatorname{grad} g(x, \xi)|$ cannot vanish on $\Gamma(t)$, hence

$$
\frac{d a}{d t}=-\oint_{\Gamma(t)} e^{u(\xi)}|\operatorname{grad} g(x, \xi)|^{-1} d s_{\xi} .
$$

(3) can be written in the following form:

$$
u(x)=-\lambda \int_{0}^{\infty} t \cdot \frac{d a}{d t} \cdot d t
$$

Integration by parts yields

$$
u(x)=\lambda \int_{0}^{\infty} a(t) d t .
$$

By the Schwarz inequality we have

$$
\oint_{\Gamma(t)} e^{u(\xi)}|\operatorname{grad} g|^{-1} d \xi \cdot \oint_{\Gamma(t)}|\operatorname{grad} g| d s_{\xi} \geqslant\left\{\oint_{\Gamma(t)} e^{u(\xi) / 2} d s_{\xi}\right\}^{2}
$$

and therefore

$$
-\frac{d a}{d t} \geqslant\left\{\oint_{\Gamma(t)} e^{u(\xi) / 2} d s_{\xi}\right\}^{2}
$$

Consider the abstract surface given by the domain $D \subset \mathbf{R}^{2}$ and the Riemann metric $d \sigma^{2}=e^{u(\xi)} d s^{2}$. Its Gaussian curvature is $K=-\Delta u /\left(2 e^{u}\right)=\lambda / 2$.

Because of the isoperimetric inequality for manifolds of constant Gaussian curvature [1, p. 514],

$$
\left\{\oint_{\Gamma(t)} e^{u(\xi) / 2} d s\right\}^{2} \geqslant 4 \pi a(t)-\frac{\lambda}{2} a^{2}(t) .
$$

This inequality together with (6) implies that

$$
-d a / d t \geqslant 4 \pi a(t)-\lambda a^{2}(t) / 2 .
$$


Thus, $m(t)=e^{-4 \pi t}(1 / a(t)-\lambda / 8 \pi)$ is a nondecreasing function of $t$. From (2) we conclude that

$$
\lim _{t \rightarrow \infty} m(t)=1 /\left(\pi R_{x}^{2} e^{u(x)}\right)
$$

Hence

$$
\begin{aligned}
& m(t) \leqslant 1 /\left(\pi R_{x}^{2} e^{u(x)}\right) \quad \text { for all } t>0, \\
& a(t) \geqslant \frac{1}{e^{4 \pi t} /\left(\pi R_{x}^{2} e^{u(x)}\right)+\lambda / 8 \pi} .
\end{aligned}
$$

If we insert this estimate into (5) and integrate, we obtain

$$
e^{u(x) / 2} \geqslant 1+\lambda R_{x}^{2} e^{u(x)} / 8 .
$$

Let us put for short $\beta=\lambda R_{x}^{2} / 8$. Then (7) yields

$$
\left[e^{u(x) / 2}-1 /(2 \beta)\right]^{2}+1 / \beta-1 /\left(4 \beta^{2}\right) \leqslant 0 .
$$

Hence, the expression $1 / \beta-1 /\left(4 \beta^{2}\right)$ must be nonpositive, and we therefore have

$$
\lambda R_{x}^{2} \leqslant 2 .
$$

From this inequality we conclude that

$$
\lambda^{*} \leqslant 2 / R_{0}^{2} \text {. }
$$

Consider now a circle of radius $R$. The radially symmetric solutions of (1) are in this case [4]

$$
u_{i}(r)=\log \frac{b_{i}}{\left(1+\lambda\left(b_{i} / 8\right) r^{2}\right)^{2}}
$$

where $r=|x|$ and

$$
b_{i}=\frac{32}{\lambda^{2} R^{4}}\left(1-\frac{\lambda R^{2}}{4}+(-1)^{i}\left(1-\frac{\lambda R^{2}}{2}\right)^{1 / 2}\right), \quad i=1,2 .
$$

the function $u_{1}(r)$ corresponds to the minimal solution [5], [2]. By [5, Theorem 3.2] it follows that Problem (1) has a solution if and only if a minimal solution exists. Thus, $\lambda^{*}=2 / R_{0}^{2}$.

We therefore have proved

THEOREM 1. Let $D$ be a simply connected domain in $\mathbf{R}^{2}$, and let $R_{0}$ be its maximal conformal radius. Then $\lambda^{*} \leqslant 2 / R_{0}^{2}$. Equality holds for the circle.

The next result is an immediate consequence of (8).

THEOREM 2. Under the assumptions of Theorem 1 we have

$$
1-\sqrt{1-4 \beta} \leqslant 2 e^{-u(x) / 2} \leqslant 1+\sqrt{1-4 \beta}
$$

where $\beta=\lambda R_{x}^{2}$. 
Equality holds at the right-hand side if $D$ is a circle, $x$ is taken at the center and $u(x)$ is the minimal solution $u_{1}(r)$. Equality holds at the left-hand side if $D$ is a circle, $x$ is taken at the center and $u(x)$ corresponds to $u_{2}(r)$.

Remark. Since $R_{x} \neq 0$ for $x \in D, x \notin \partial D$, (11) leads to the conjecture that for fixed $\lambda$ all solutions of Problem (1) are uniformly bounded.

Let $d(x)$ be the distance from the point $x \in D$ to the boundary $\partial D$. By the monotony of $R_{x}$ with respect to the domain it follows that $R_{x} \geqslant d(x)$. This inequality together with (11) leads to the

COROLlARY. Under the assumptions of Theorem 1 we have

$$
1-\sqrt{1-\lambda d^{2}(x) / 2} \leqslant 2 e^{-u(x) / 2} \leqslant 1+\sqrt{1-\lambda d^{2}(x) / 2} .
$$

\section{REFERENCES}

1. A. D. Aleksandrov, Intrinsic geometry of convex surfaces, OGIZ, Moscow, 1948; German transl., Die innere Geometrie der konvexen Flächen, Akademie-Verlag, 1955. MR 10, 619; 17, 74.

2. C. Bandle, Existence theorems, some qualitative results and a priori bounds for a class of nonlinear Dirichlet problems, Arch. Rational Mech. Anal. 58 (1975), 219-238.

3. M. G. Crandall and P. H. Rabinowitz, Some continuation and variational methods for positive solutions of nonlinear elliptic eigenvalue problems, Arch. Rational Mech. Anal. 58 (1975), 207-218.

4. I. M. Gelfand, Some problems in the theory of quasi-linear equations, Uspehi Mat. Nauk 14 (1959), no. 2 (86), 87-158; English transl., Amer. Math. Soc. Transl. (2) 29 (1963), 295-381. MR 22 \# 1736; 27 \#3921.

5. H. B. Keller and D. S. Cohen, Some positive problems suggested by nonlinear heat generation, J. Math. Mech. 16 (1967), 1361-1376. MR 35 \#4552.

6. G. Pólya and G. Szegö, Isoperimetric inequalities in mathematical physics, Ann. of Math. Studies, no. 27, Princeton Univ. Press, Princeton, N. J., 1951. MR 13, 270.

Mathematisches Institut, Universität BaSel, CH-4051 Basel, Switzerland 